\title{
Improving Longitudinal Transversal Relaxation Of Gadolinium Chelate Using Silica Coating Magnetite Nanoparticles
}

This article was published in the following Dove Press journal: International Journal of Nanomedicine

\author{
Kai Xu (D) ${ }^{1,2}$ \\ Heng Liu ${ }^{3}$ \\ Junfeng Zhang ${ }^{1,2}$ \\ Haipeng Tong ${ }^{1,2}$ \\ Zhenghuan Zhao ${ }^{4}$ \\ Weiguo Zhang ${ }^{1,2}$
}

'Department of Radiology, Daping Hospital, Army Medical Center of PLA, Army Medical University, Chongqing 400042, People's Republic of China; ${ }^{2}$ Chongqing Clinical Research Center for Imaging and Nuclear Medicine,

Chongqing 400042, People's Republic of China; ${ }^{3}$ Department of Radiology, PLA

Rocket Force Characteristic Medical Center, Beijing 100088, People's Republic of China; ${ }^{4}$ Department of Pharmaceutical Engineering, College of Pharmaceutical Sciences, Southwest University, Chongqing 400716, People's Republic of China

Correspondence: Zhenghuan Zhao Department of Pharmaceutical Engineering, College of Pharmaceutical Sciences, Southwest University, No. 2 Tiansheng Road, Beibei District, Chongqing 400715, People's Republic of China

Tel +86236875 7621

Fax +86 236875 7620

Email roddirck@swu.edu.cn

Weiguo Zhang

Department of Radiology, Daping

Hospital, I0th Changjiang Road, Yuzhong

District, Chongqing 400042, People's

Republic of China

Tel +86236875 7621

Fax +86 236875 7620

Email wgzhang0I@163.com
Introduction and objective: Precisely and sensitively diagnosing diseases especially early and accurate tumor diagnosis in clinical magnetic resonance (MR) scanner is a highly demanding but challenging task. Gadolinium $(\mathrm{Gd})$ chelate is the most common $T_{1}$ magnetic resonance imaging (MRI) contrast agent at present. However, traditional Gd-chelates are suffering from low relaxivity, which hampers its application in clinical diagnosis. Currently, the development of nano-sized Gd based $T_{1}$ contrast agent, such as incorporating gadolinium chelate into nanocarriers, is an attractive and feasible strategy to enhance the $T_{1}$ contrast capacity of Gd chelate. The objective of this study is to improve the $T_{1}$ contrast ability of Gdchelate by synthesizing nanoparticles (NPs) for accurate and early diagnosis in clinical diseases.

Methods: Reverse microemulsion method was used to coat iron oxide (IO) with tunable silica shell and form cores of NPs IO@SiO $\mathrm{Si}_{2}$ at step one, then Gd-chelate was loaded on the surface of silica-coated iron oxide NPs. Finally, Gd-based silica coating magnetite NPs $\mathrm{IO} @ \mathrm{SiO}_{2}$-DTPA-Gd was developed and tested the ability to detect tumor cells on the cellular and in vivo level.

Results: The $r_{1}$ value of IO@ $\mathrm{SiO}_{2}$-DTPA-Gd NPs with the silica shell thickness of $12 \mathrm{~nm}$ was about $33.6 \mathrm{mM}^{-1} \mathrm{~s}^{-1}$, which was approximately 6 times higher than Gd-DTPA, and based on its high $T_{1}$ contrast ability, $\mathrm{IO} @ \mathrm{SiO}_{2}$-DTPA-Gd NPs could effectively detect tumor cells on the cellular and in vivo level.

Conclusion: Our findings revealed the improvement of $T_{1}$ relaxation was not only because of the increase of molecular tumbling time caused by the $\mathrm{IO} @ \mathrm{SiO}_{2}$ nanocarrier but also the generated magnetic field caused by the IO core. This nanostructure with high $T_{1}$ contrast ability may open a new approach to construct high-performance $T_{1}$ contrast agent.

Keywords: gadolinium chelate, silica, iron oxide, nanoparticles, $T_{1}$ relaxivity, tumbling time

\section{Introduction}

Magnetic resonance imaging has been widely used in clinical diagnosis due to its high spatial and temporal resolution, non-invasive and non-radioactive imaging. ${ }^{1,2}$ However, its sensitivity and specificity are insufficient to provide enough signal in clinical MR scanner to achieve the diagnosis, especially early and accurate tumor diagnosis. Thus, various MRI contrast agents have been developed, such as gadolinium-based, ${ }^{3,4}$ iron-based, ${ }^{5-7}$ and manganese-based agents, ${ }^{8-11}$ to improve its accuracy and sensitivity. Compared to iron or manganese element, gadolinium exhibits long electronic relaxation time and more unpaired electrons. These advantages endow Gdchelates to be the most common $T_{1}$ MRI contrast agent to shorten longitudinal 
relaxation time of protons and assist cancer diagnosis in clinical. Currently, different Gd-chelates have been approved by the Food and Drug Administration (FDA) for clinical imaging, such as Gd-DTPA (Magnevist) and Gd-DOTA (Dotarem). However, Gd-chelates are suffering from low relaxivity and contrast efficiency, which hamper the application on clinical diagnosis. Based on the classical SolomonBloembergen-Morgan (SBM) theory, the $T_{1}$ relaxivity is determined by a few parameters, including proton residence lifetime, molecular tumbling time and the number of coordinating. ${ }^{12,13}$ In theory, along with the increase of molecular tumbling time, number of coordinating, and decrease of proton residence lifetime, the $T_{1}$ contrast capacity of contrast agent is improved. Nano-sized materials exhibit significantly slower molecular tumbling than small molecules, which could improve $T_{1}$ MRI contrast ability of Gdbased agent. ${ }^{14-16}$ Thus, development of nano-sized Gd based $T_{1}$ contrast agent, such as incorporating Gd-chelates into nanocarriers, is an attractive and feasible strategy to enhance the $T_{1}$ contrast capacity of Gd-chelates.

On the basis of its high biocompatibility and $T_{2}$ contrast capacity, superparamagnetic iron oxide NPs have been used as the nanocarrier for constructing $T_{1} / T_{2}$ dualmodal MRI contrast agent to achieve tumor diagnosis. ${ }^{17-19}$ However, the effect of iron oxide NPs which could affect the $T_{1}$ contrast ability of Gd-chelate was ignored. Previous research indicated that the magnetic field generated by superparamagnetic $T_{2}$ contrast agent might disturb the relaxation process of proton caused by the $T_{1}$ contrast agent, which may quench the acceleration effect of Gdchelate to proton relaxation. ${ }^{20-22}$ It should be noted that this quenching effect decreases with the increase of the distance between $T_{2}$ contrast agent and $T_{1}$ contrast agent. Meanwhile, the magnetic field induced by the $T_{2}$ contrast agent may result in $T_{1}$ spin alignment in the same direction, which lead to the enhancement of $T_{1}$ effect. $^{23-26}$ Thus, the distance between $T_{2}$ and $T_{1}$ contrast agent may determine the effect of $T_{2}$ contrast agent to a $T_{1}$ contrast agent. The Strategy of loading Gd-chelate on the surface of iron oxide NPs with suitable distance may develop a new $T_{1}$ contrast agent with high performance.

Recently, silica coating has been widely used to improve the biocompatibility and stability of biomedical materials. ${ }^{27-29}$ Among all approaches, the reverse microemulsion method has been widely used to coat hydrophobic NPs with tunable silica shell. ${ }^{30,31}$ Furthermore, the silica coating shell is easy to couple and label functional molecules based on the abundant functional group. $^{32,33}$ These unique features endow it to be the best tool to adjust the distance between iron oxide NPs and Gdchelate and discuss the distance effect on $T_{1}$ contrast ability of Gd-chelate. Herein, we developed a strategy to improve the $T_{1}$ contrast ability of Gd-chelate by loading the Gd-chelate on the surface of silica-coated iron oxide NPs (IO@ $\mathrm{SiO}_{2}$-DTPA-Gd). The $r_{1}$ value of IO@SiO $\mathrm{SiO}_{2}$-DTPA-Gd NPs with the silica shell thickness of $\sim 12 \mathrm{~nm}$ was about $33.6 \mathrm{mM}^{-1} \mathrm{~s}^{-1}$, which was approximately 6 times higher than Gd-DTPA. Further analysis indicated that the improvement of $T_{1}$ relaxation was not only because of the increase of molecular tumbling time caused by the $\mathrm{IO} @ \mathrm{SiO}_{2}$ nanocarrier but also the generated magnetic field caused by the $\mathrm{IO}$ core. In addition, the improvement effect of $T_{1}$ relaxation increased with the growth of silica shell thickness. Based on its high $T_{1}$ contrast ability, $\mathrm{IO} @ \mathrm{SiO}_{2}-$ DTPA-Gd NPs can effectively detect tumor cells on the cellular and in vivo level. This nanostructure with high $T_{1}$ contrast ability may open a new approach to construct high-performance $T_{1}$ contrast agent.

\section{Materials And Methods \\ Materials}

Oleic acid (tech 90\%), tetraethylorthosilicate (TEOS 99.9\%), (3-aminopropyl) triethoxysilane (APTES 97\%), 1-octadecene (90\%), and oleic acid (90\%) were purchased from Alfa Aesar. (Shanghai, China); p-SCN-Bn-DTPA was purchased from Macrocyclics; Sodium oleate, iron chlorides, hexane, isopropanol, ammonium hydroxide, and ethanol were purchased from Sinopharm Chemical Reagent Co. Ltd. (Shanghai, China). All chemicals were used as received without further purification.

\section{Characterizations}

Transmission electron microscopy (TEM) images were taken on JEOL JEM-2100 at $200 \mathrm{kV}$. The X-ray diffraction (XRD) patterns were obtained on the Rigaku Ultima IV system. The iron and gadolinium concentrations in NPs were measured with inductively coupled plasma atomic emission spectroscopy (ICP-AES). The absorbance was measured using a microplate reader (MultiSkan FC microplate reader, Thermo scientific). The MRI testing and $T_{1}$ relaxation time measurements were tested at a $0.5 \mathrm{~T}$ NMR120-Analyst NMR Analyzing\&Imaging system (Niumag Corporation, Shanghai, China).

\section{Preparation Of Iron Oxide NPs}

In a typical experiment, $0.8 \mathrm{~g}$ iron-oleate $(0.88 \mathrm{mmol})$ synthesized as aforementioned and $142 \mu \mathrm{L}$ oleic acid $(0.44 \mathrm{mmol})$ 
were dissolved in $12 \mathrm{~mL} \mathrm{1-octadecene} \mathrm{at} \mathrm{room} \mathrm{temperature.}$ The mixture was degassed in vacuum for $30 \mathrm{~min}$ and backfilled with argon to remove any low volatile impurities and oxygen at room temperature. After that, the reaction solution was heated to reflux with a constant heating rate of $3.3^{\circ} \mathrm{C} \min ^{-1}$ and kept at that temperature for $1 \mathrm{~h}$. The resultant solution was then cooled to room temperature and mixed with $30 \mathrm{~mL}$ isopropanol to precipitate the NPs. The NPs were separated by centrifugation and washed three times with ethanol. After washing, the NPs were dissolved in hexane for long term storage at $4{ }^{\circ} \mathrm{C}$.

\section{Preparation Of $\mathrm{IO} @ \mathrm{SiO}_{2}-\mathrm{NH}_{2} \mathrm{NPs}$}

The reverse microemulsion method was used to prepare $\mathrm{IO} @ \mathrm{SiO}_{2}-\mathrm{NH}_{2} \mathrm{NPs}$. In a typical experiment, we added 1.2 $\mathrm{mL}$ of Co-520, $2 \mathrm{~mL}$ of iron oxide NPs solution $(0.8 \mathrm{mg} / \mathrm{mL})$, $200 \mu \mathrm{L}$ of TEOS, and $400 \mu \mathrm{L}$ of ammonia into $20 \mathrm{~mL}$ of cyclohexane. After $16 \mathrm{~h}$ reaction at room temperature, we added $20 \mu \mathrm{L}$ of APTES to modify the amino group on the surface. The resultant solution was mixed with $40 \mathrm{~mL}$ of ethanol to precipitate the nanomaterials at $14,000 \mathrm{rpm}$. The nanomaterials were washed three times with ethanol. After washing, this nanomaterial was dissolved in ultrapure water $(18.2 \mathrm{M} \Omega \cdot \mathrm{cm})$ at room temperature for further use. By adjusting the amount of TEOS, the IO@ $\mathrm{SiO}_{2}-\mathrm{NH}_{2} \mathrm{NPs}$ with different shell thicknesses could be synthesized.

\section{Conjugation Of DTPA-Gd On IO@SiO ${ }^{-}$ $\mathrm{NH}_{2} \mathrm{NPs}$}

The conjugation of DTPA-Gd was achieved by reacting the IO@ $\mathrm{SiO}_{2}-\mathrm{NH}_{2}$ NPs with p-SCN-DTPA with the molar ratio of $1: 3$. The NPs were separated by centrifugation and washed three times with water to remove the free $\mathrm{p}-\mathrm{SCN}$ DTPA. After washing, the NPs were dissolved in $10 \mathrm{~mL}$ $\mathrm{GdCl}_{3} \cdot 6 \mathrm{H}_{2} \mathrm{O}(194.7 \mathrm{mg})$ solution $(\mathrm{pH}$ 7.4) and stirred overnight. The resultant product was centrifuged and redispersed in water three times and dissolved in water for long term storage at $4{ }^{\circ} \mathrm{C}$.

\section{Measurement Of MR Relaxivities Of NPs}

To measure the $T_{1}$ relaxivities, samples with different gadolinium ion concentrations were dispersed in $1 \%$ agarose solution. The $T_{1}$ relaxation times for all the samples were measured by a 0.5 T NMI20-Analyst NMR system and used to calculate the relaxation rates of the samples. The $T_{1^{-}}$ weighted MRI images for the samples were acquired using the MSE sequence as the following parameters: TR/TE $=100 /$ $12 \mathrm{~ms}, 256 \times 256$ matrices, thickness $=1 \mathrm{~mm}, \mathrm{NS}=2$.

\section{Cell Culture}

HeLa cells were purchased from the Cell Bank of Chinese Academy of Sciences (Shanghai, China). HeLa cells were cultured in Dulbecco's Modified Eagle's Medium (DMEM medium) supplemented with $10 \%$ fetal bovine serum (FBS, Hyclone) and antibiotics $(100 \mathrm{mg} / \mathrm{mL}$ streptomycin and $100 \mathrm{U} / \mathrm{mL}$ penicillin) and maintained in a humidified atmosphere of $5 \% \mathrm{CO}_{2}$ at $37{ }^{\circ} \mathrm{C}$.

\section{Cytotoxicity Evaluation}

Cells were seeded into a 96-well plate with a density of $5 \times 10^{3}$ cells/well in the culture medium and incubated in the atmosphere of $5 \% \mathrm{CO}_{2}$ at $37{ }^{\circ} \mathrm{C}$ for $12 \mathrm{~h}$. The cells were then incubated with IO@ $\mathrm{SiO}_{2}$-DTPA-Gd NPs at a serial of Gd concentrations for $24 \mathrm{~h}$. Each experiment in the same concentration was performed in five times. Subsequently, the culture medium was removed, we replaced the growth medium with DMEM containing $0.5 \mathrm{mg} / \mathrm{mL}$ of 3-(4, 5-dimethylthiazol-2yl)-2, 5-diphenyltetrazolium bromide (MTT) and incubated for another $4 \mathrm{~h}$ at $37^{\circ} \mathrm{C}$. After discarding the culture medium, $100 \mu \mathrm{L}$ of DMSO was added to dissolve the precipitates and the resulting solution was measured for absorbance at $492 \mathrm{~nm}$ using a MultiSkan FC microplate reader (Thermo scientific).

\section{Cellular Imaging}

HeLa cells were seeded with a density of $5 \times 10^{3}$ cells/well in the culture medium and incubated in the atmosphere of 5\% $\mathrm{CO}_{2}$ at $37^{\circ} \mathrm{C}$ for $12 \mathrm{~h}$. The cells were then incubated with $\mathrm{IO} @ \mathrm{SiO}_{2}$-DTPA-GdNPs and DTPA-Gd for $6 \mathrm{~h}$. Each experiment in the same concentration was performed in three wells. We then centrifuged the cells at $200 \mathrm{~g}$ for $5 \mathrm{~min}$ to harvest them. Then, we concentrated the cells at the button of the EP tube by centrifugation and performed $T_{1}$-weighted MRI imaging on a $0.5 \mathrm{~T}$ NMI20-Analyst NMR system. The samples were scanned using a multi-echo $T_{1}$-weighted fast spin-echo imaging sequence $(\mathrm{TR} / \mathrm{TE}=100 / 12 \mathrm{~ms}, 256 \times 256$ matrices, thickness $=1 \mathrm{~mm}, \mathrm{NS}=16$ ).

\section{In Vivo MR Imaging}

For establishment of HeLa tumor model, female Balb/c nude mice (25 $\pm 2 \mathrm{~g}, 4-5$ weeks) were supplied by Center of Experimental Animals, Daping Hospital, China. All animal experiments were executed according to the protocol approved by the Animal Care and Use Committee of Army Medical University, China. Xenografted tumor models were made by subcutaneous inoculation of $10^{6} \mathrm{HeLa}$ cells suspended in $100 \mu \mathrm{L}$ PBS at the right back of mice. When the 
tumor reached $100 \mathrm{~mm}^{3}$, the mice bearing tumor were intravenously injected IO@ $\mathrm{SiO}_{2}$-DTPA-Gd NPs $(2 \mathrm{mg} \mathrm{Gd} / \mathrm{kg}$ body weight). The $T_{1}$-weighted MRI imaging was performed on the $7 \mathrm{~T}$ Animal MRI (Bruker) and the MR images were acquired using the following parameters: $\mathrm{TR} / \mathrm{TE}=1500 / 8 \mathrm{~ms}$, $256 \times 256$ matrices, thickness $=1 \mathrm{~mm}, \mathrm{FOV}=250 \times 250 \mathrm{~mm}$. The MR images were sequentially acquired at pre-injection and $15,30,45,60$ and 120 min post-injection.

\section{Statistical Analysis}

Statistical analysis was performed using the Student's $t$ test for unpaired data, $\mathrm{p}$ values of less than 0.05 were
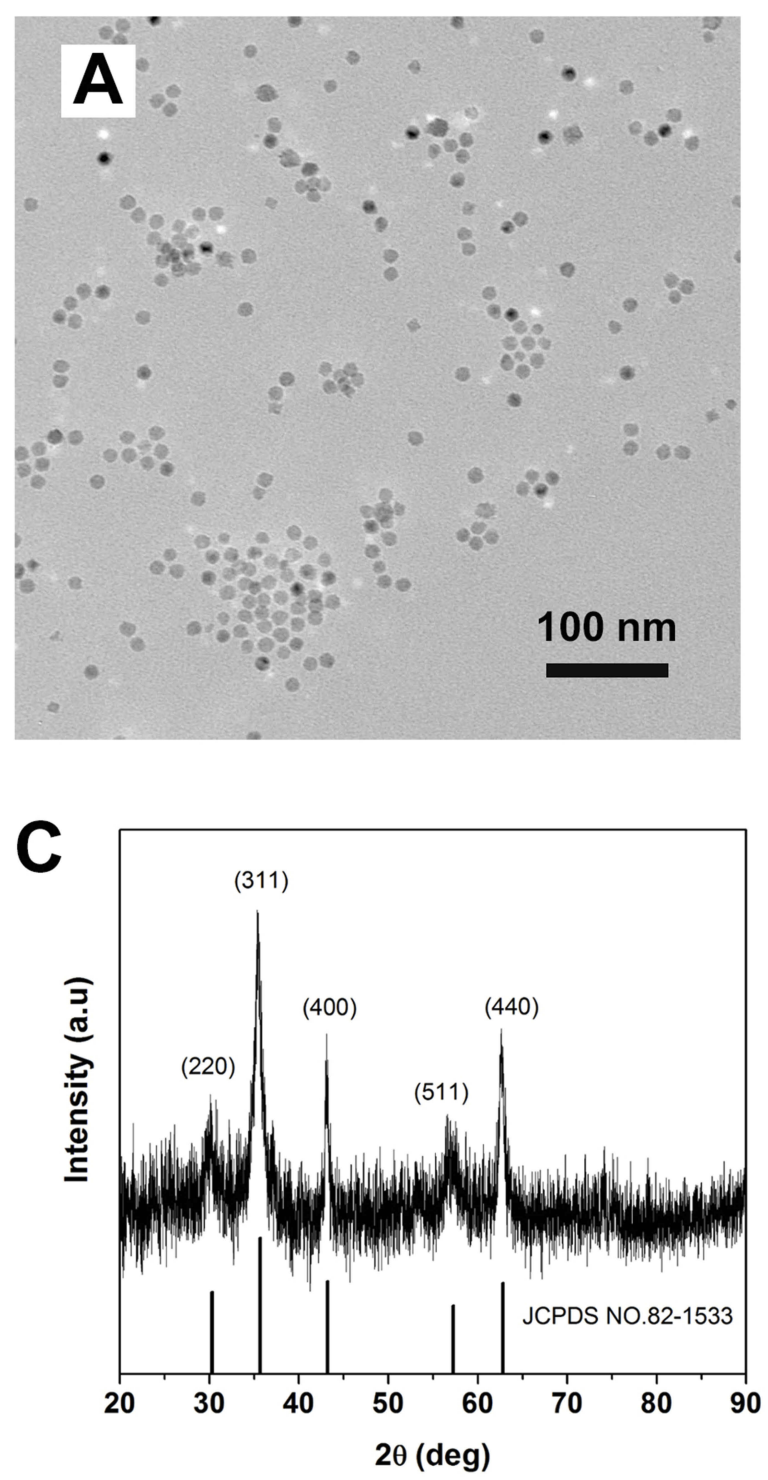

accepted as a statistically significant difference compared to controls.

\section{Results And Discussion \\ Synthesis And Characterization}

To obtain silica-coated iron oxide nanostructure to improve the $T_{1}$ relaxation of Gd-chelate, $\mathrm{Fe}_{3} \mathrm{O}_{4}$ (IO) NPs were synthesized by thermal decomposition of iron-oleate in 1-octadecene. TEM images (Figure 1A) indicated that the as-prepared product was monodispersed spherical NPs in high yield. The diameters of these products were about $12 \mathrm{~nm}$. Additionally, the high-resolution TEM (HRTEM)
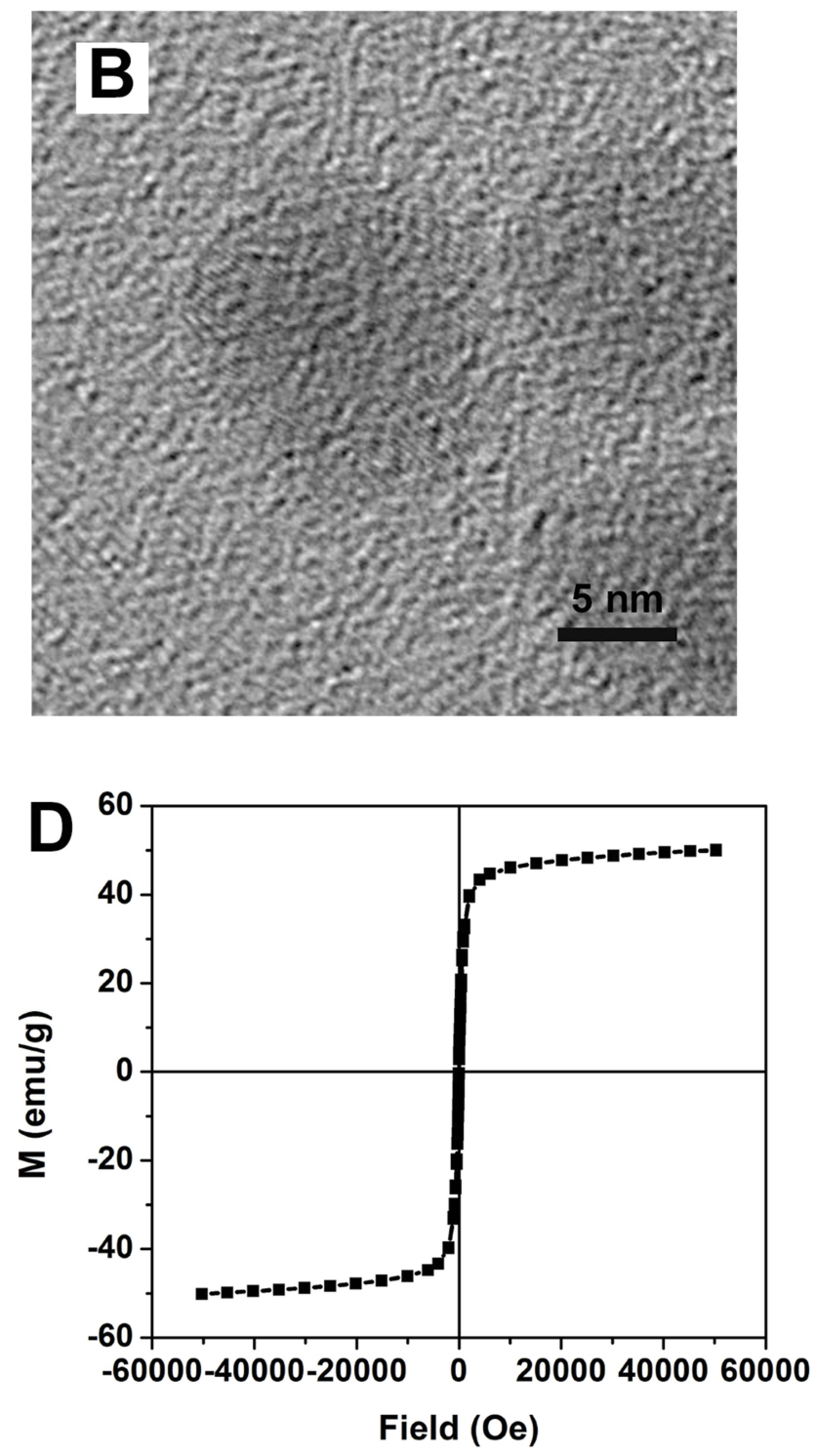

Figure I Characteristic of IO nanoparticles. (A) TEM and (B) HRTEM images of $\mathrm{Fe}_{3} \mathrm{O}_{4}$ nanoparticles. (C) XRD analysis of IO nanoparticles, indicating the typical magnetite diffractogram pattern. (D) M-H curve of 10 .

Abbreviations: IO, iron oxide; TEM, transmission electron microscopy; HRTEM, high-resolution TEM; XRD, X-ray diffraction. 
images (Figure 1B) showed the uniform lattice fringes across the whole NPs, revealing its good crystallinity and implying its good magnetic property. Further X-ray powder diffraction pattern analysis (Figure 1C) confirmed that the product exhibited the typical IO diffractogram pattern (JCPDS number 82-1533). We then analyzed the magnetic properties of as-prepared IO NPs by the superconducting quantum interference device magnetometer at $300 \mathrm{~K}$. It appeared that the IO NPs showed a smooth $M-H$ curve (Figure 1D) with no hysteresis at ambient temperature, suggesting the superparamagnetic behavior. Moreover, the $M_{\mathrm{s}}$ value of IO NPs was about $49 \mathrm{emu} / \mathrm{g}$. These magnetic features endowed IO NPs to generate the magnetic field under the local filed to affect the $T_{1}$ relaxation of Gd-chelate.

We further coated the IO NPs by silica shell through a reverse microemulsion method with TEOS and (3-aminopropyl) APTES (Figure 2A). TEM images showed the typical spherical core/shell structure with the average shell thickness of $\sim 12 \mathrm{~nm}$ (Figure 2B). Moreover, all product showed uniform coating of silica on IO NPs with single core and no core-free silica NPs existed. These features ensured it to be the suitable candidate for coupling Gd-chelate to construct new $T_{1}$ contrast agent with uniform ability. In addition, the morphology and size of single-core IO NPs maintained the same before and after coating (Figure $2 \mathrm{~B}$ and $\mathrm{C}$ ), enabling $\mathrm{IO} @ \mathrm{SiO}_{2}$ with magnetic ability to affect the $T_{1}$ relaxation of Gdchelate. The conjugation of Gd-chelate on the surface of $\mathrm{IO} @ \mathrm{SiO}_{2}$ was achieved through the combination between amino groups on $\mathrm{IO} @ \mathrm{SiO}_{2}$ and isothiocyanate of DTPA. ICP-AES analyses indicated that the $\mathrm{Gd}$ to $\mathrm{Fe}$ ratio is about 0.1:1. The TEM image showed that the IO@ $\mathrm{SiO}_{2-}$ DTPA-Gd NPs exhibited similar size and morphology to that of $\mathrm{IO} @ \mathrm{SiO}_{2}$ NPs (Figure 2C and D). Furthermore, the $\mathrm{IO} @ \mathrm{SiO}_{2}$-DTPA-Gd NPs showed high colloidally stable in water at room temperature, which was essential for further biological application.

\section{MR Relaxivities Investigation}

To evaluate the MRI performance of IO@SiO $\mathrm{SiO}_{2}-\mathrm{DTPA}-\mathrm{Gd}$ NPs, we detected its $T_{1}$ relaxivity by a $0.5 \mathrm{~T}$ MRI scanner. In
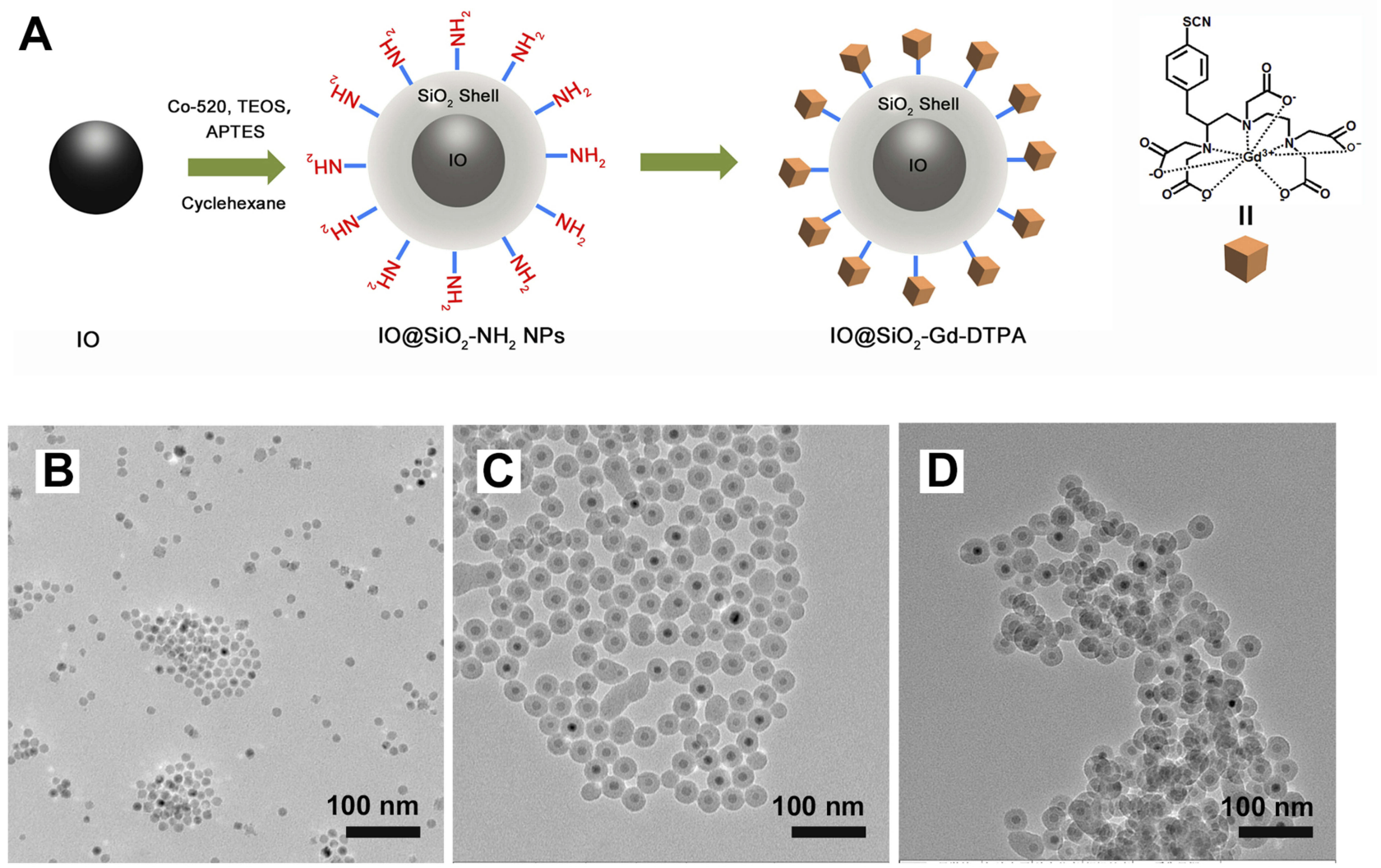

Figure 2 Synthesis of $1 \mathrm{O} @ \mathrm{SiO}_{2}$-DTPA-Gd NPs. (A) schematic cartoon illustrates the synthesis of $1 \mathrm{O} @ \mathrm{SiO}_{2}$-DTPA-Gd. TEM images of (B) $1 \mathrm{O}$ core, (C) IO@SiO $10 @ S_{2}$-DTPA-Gd.

Abbreviations: Gd, gadolinium; IO, iron oxide; TEM, transmission electron microscopy; NPs, nanoparticles. 
addition, DTPA-Gd was used for comparison (Figure 3A). $T_{1}$ relaxation analyses indicated that $\mathrm{IO} @ \mathrm{SiO}_{2}$-DTPA-Gd NPs showed significantly higher $T_{1}$ contrast ability than free DTPA-Gd (Figure 3B and C). The $r_{1}$ value of $\mathrm{IO} @ \mathrm{SiO}_{2}-$ DTPA-Gd NPs was approximately $33.6 \mathrm{mM}^{-1} \mathrm{~s}^{-1}$ (Figure S1), which was about 6 times higher than that of DTPA-Gd $\left(5.5 \mathrm{mM}^{-1} \mathrm{~s}^{-1}\right)$. Consistent with the $r_{1}$ values analyses, IO@ $\mathrm{SiO}_{2}$-DTPA-Gd NPs exhibited better $T_{1}$ contrast imaging ability than free DTPA-Gd at the same concentration of Gd. Since the $\mathrm{IO} @ \mathrm{SiO}_{2}$ showed negligible $T_{1}$ contrast ability (Figure S2), the improved $T_{1}$ contrast ability could be mainly ascribed to the improvement of molecular tumbling time of DTPA-Gd caused by the nanocarrier. ${ }^{34,35}$ Additionally, the magnetic field induced by the $T_{2}$ contrast agent may also result in $T_{1}$ spin alignment in the same direction and enhancement of $T_{1}$ effect. To investigate whether the magnetic $\mathrm{IO} @ \mathrm{SiO}_{2}$ core could improve the $T_{1}$ relaxation of DTPA-Gd, we synthesized $\mathrm{SiO}_{2}$-DTPA-Gd with similar size as the control group (Figure 4A). TEM analyses showed that $\mathrm{SiO}_{2}$-DTPA-Gd exhibited a similar size to IO@SiO ${ }_{2}$-DTPA-Gd NPs (Figure 4B), implying the similar molecular tumbling time of $\mathrm{SiO}_{2}$-DTPA-Gd and IO@ $\mathrm{SiO}_{2}$-DTPA-Gd NPs. These results could exclude the effect of molecular tumbling time on further $T_{1}$ relaxation analyses of $\mathrm{SiO}_{2}$-DTPA-Gd and $\mathrm{IO} @ \mathrm{SiO}_{2}$-DTPA-Gd NPs. We detected the $T_{1}$ relaxation of $\mathrm{SiO}_{2}$-DTPA-Gd and IO@ $\mathrm{SiO}_{2}$-DTPA-Gd NPs by a 0.5 T MRI scanner (Figure $4 \mathrm{C}$ and $\mathrm{D}$ ). Interestingly, it appeared that $\mathrm{SiO}_{2}-$ DTPA-Gd $\left(22.1 \mathrm{mM}^{-1} \mathrm{~s}^{-1}\right)$ showed notable higher $T_{1}$ relaxation than DTPA-Gd $\left(5.5 \mathrm{mM}^{-1} \mathrm{~s}^{-1}\right)$, which could be attributed to the increase of molecular tumbling time (Figure S3). It's worth note that the $r_{1}$ value of $\mathrm{IO} @ \mathrm{SiO}_{2}$ DTPA-Gd NPs was $33.6 \mathrm{mM}^{-1} \mathrm{~s}^{-1}$, which was significantly higher than that of $\mathrm{SiO}_{2}$-DTPA-Gd. Considering that IO@ $\mathrm{SiO}_{2}$-DTPA-Gd and $\mathrm{SiO}_{2}$-DTPA-Gd NPs had the similar molecular tumbling time, these results clearly indicated
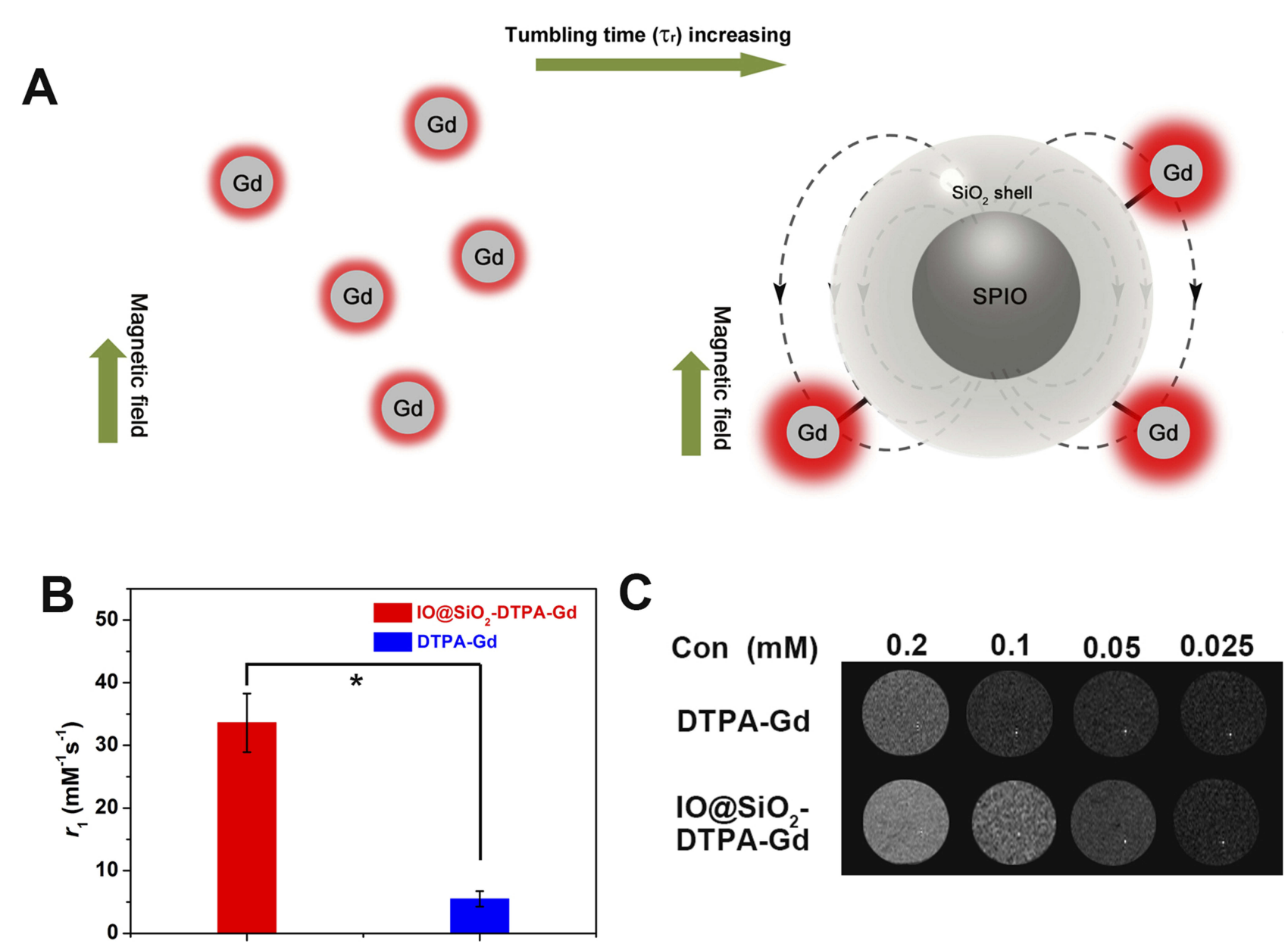

Figure $3 T_{1}$ relaxation analysis of $10 @ S_{S i O}-\mathrm{DTPA}-\mathrm{Gd}$ NPs. (A) Schematic cartoons illustrate the increased molecular tumbling time result in the improve of IO@SiO ${ }_{2}-$ DTPA-Gd $T_{1}$ relaxation compared to DTPA-Gd. (B) $T_{1}$ relaxation analyses of $1 \mathrm{O} @ S \mathrm{SiO}_{2}$-DTPA-Gd and DTPA-Gd, $p<0.05(*)$. (C) $T_{1}$-weighted images of DTPA-Gd and $1 \mathrm{O} @ \mathrm{SiO}_{2}$-DTPA-Gd at different concentration.

Abbreviations: Gd, gadolinium; IO, iron oxide; NPs, nanoparticles. 
A
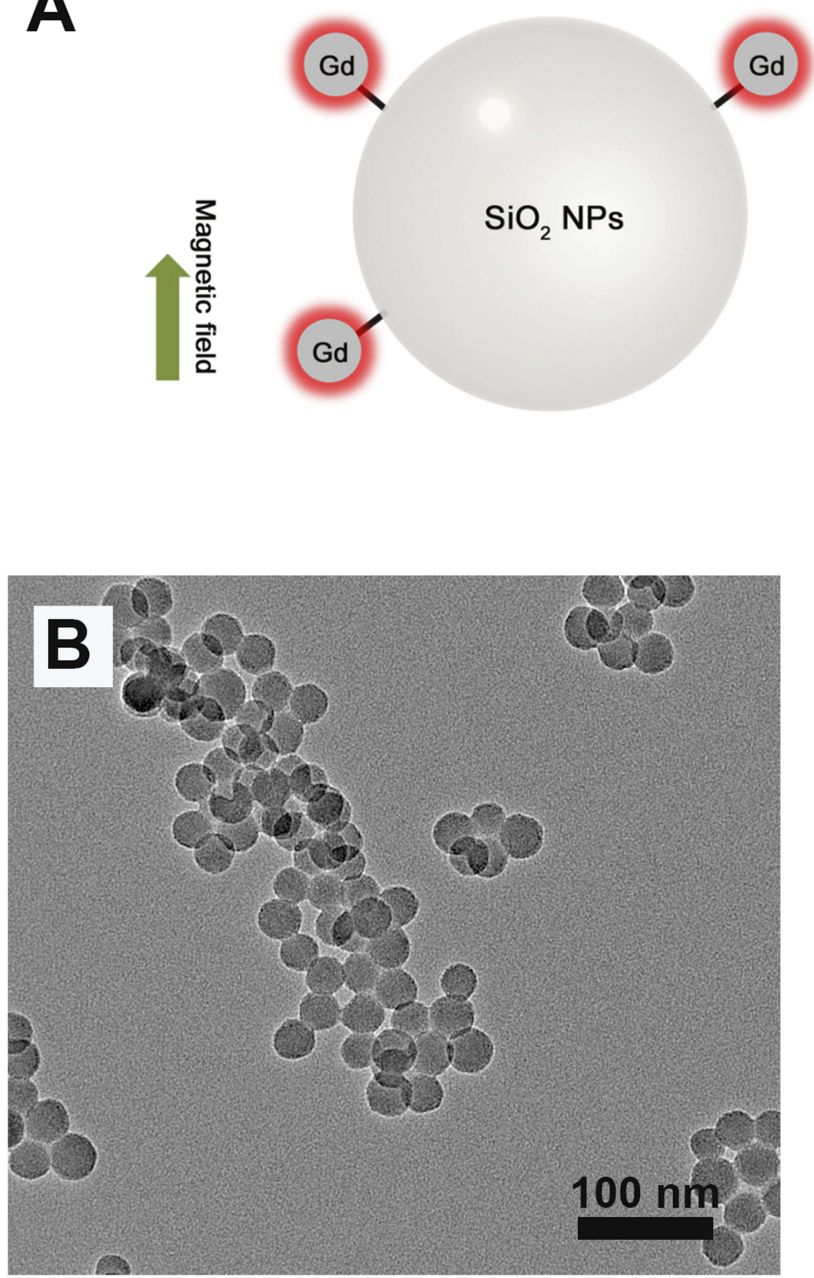
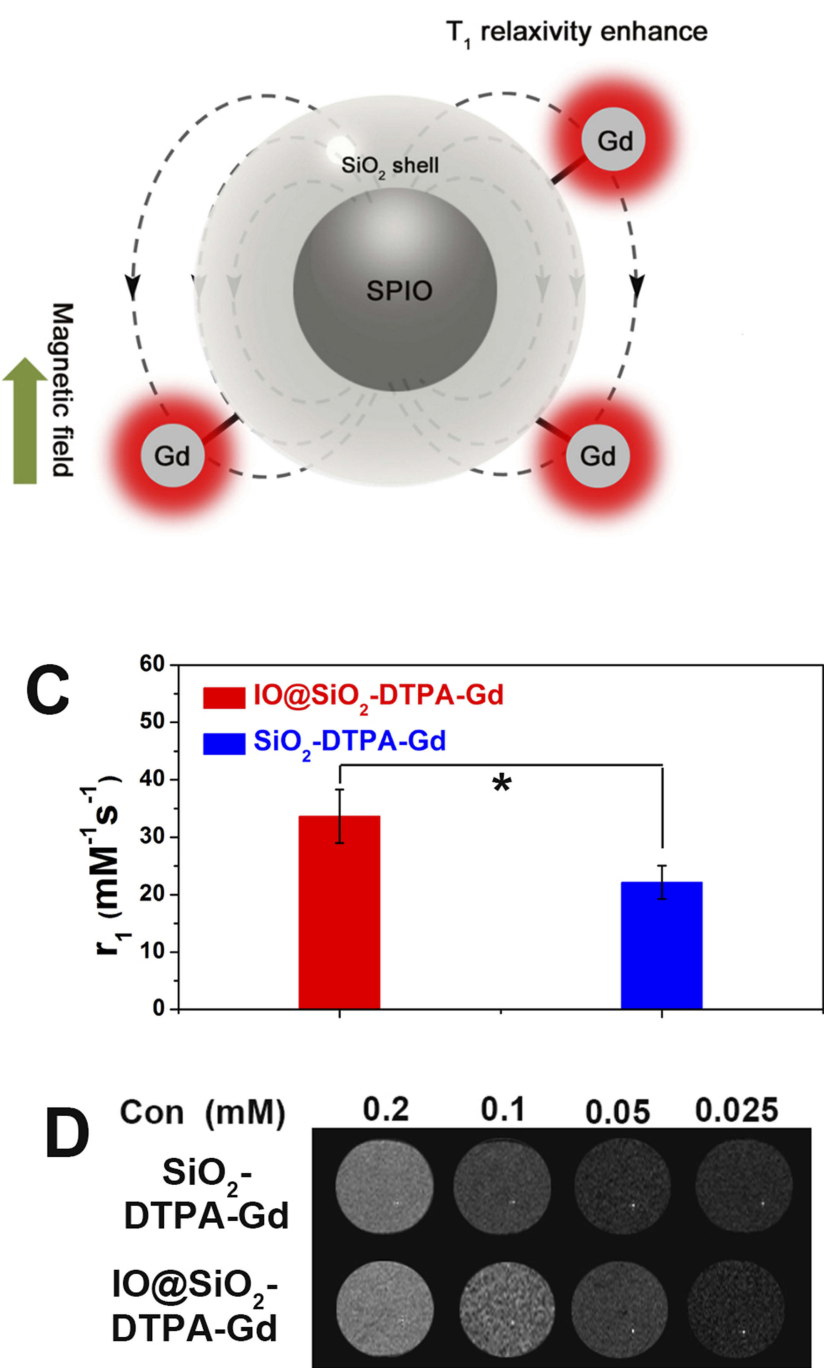

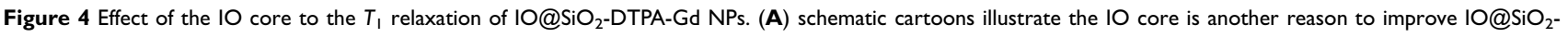
DTPA-Gd NPs $T_{1}$ relaxation. (B) TEM images of $\mathrm{SiO}_{2}$-DTPA-Gd with the similar size to IO@SiO ${ }_{2}-\mathrm{DTPA}-\mathrm{Gd}$ NPs. (C) $T_{1}$ relaxation analyses of IO@SiO - -DTPA-Gd and $\mathrm{SiO}_{2}$-DTPA-Gd NPs, $p<0.05(*)$. (D) $T_{1}$-weighted images of $\mathrm{SiO}_{2}$-DTPA-Gd and $10 @ \mathrm{SiO}_{2}$-DTPA-Gd NPs at different concentration.

Abbreviations: Gd, gadolinium; IO, iron oxide; TEM, transmission electron microscopy, NPs, nanoparticles.

that the improved $T_{1}$ relaxation of $\mathrm{IO} @ \mathrm{SiO}_{2}-\mathrm{DTPA}-\mathrm{Gd} \mathrm{NPs}$ was not only caused by the increased molecular tumbling time but also caused by the magnetite core of $\mathrm{IO} @ \mathrm{SiO}_{2}$. Since the increments caused by the increase of molecular tumbling time was larger than that of introducing of magnetite, molecular tumbling time increase was the main reason to elevate the $r_{1}$ value of $\mathrm{IO} @ \mathrm{SiO}_{2}$-DTPA-Gd NPs. Further $T_{1}$-weighted photon images revealed that the $T_{1}$ signal increases of $\mathrm{IO} @ \mathrm{SiO}_{2}$-DTPA-Gd NPs were more obvious than that of $\mathrm{SiO}_{2}$-DTPA-Gd, which endowed it with the ability to be a potential candidate for disease diagnosis.

We further assessed the effect of the $\mathrm{SiO}_{2}$ thickness on the $T_{1}$ contrast ability of IO@ $\mathrm{SiO}_{2}$-DTPA-Gd NPs (Figure 5A). We synthesized IO@ $\mathrm{SiO}_{2}$-DTPA-Gd NPs with the silica thickness of 0,5 , and $12 \mathrm{~nm}$ by reverse microemulsion method through tuning the amount of TEOS. TEM images indicated that the thickness of the silica shell increased with the increase of TEOS ratio (Figure 5B and D). More importantly, all products showed the single core structure without core-free silica nanosphere. This feature endowed these products as suitable samples to discuss the effect of silica shell thickness to $T_{1}$ relaxation of $\mathrm{IO} @ \mathrm{SiO}_{2}$-DTPA-Gd NPs. $T_{1}$ relaxation analyses indicated that the $r_{1}$ values of $\mathrm{IO} @ \mathrm{SiO}_{2^{-}}$ DTPA-Gd NPs with a thickness of 0,5 , and $12 \mathrm{~nm}$ were 9.6, 14.8 , and $33.6 \mathrm{mM}^{-1} \mathrm{~s}^{-1}$, respectively (Figure 5E and F). These results revealed that the $T_{1}$ contrast ability of $\mathrm{IO} @ \mathrm{SiO}_{2}$-DTPA-Gd increased with the increase of shell thickness. It should be noted that IO@SiO $\mathrm{SiO}_{2} \mathrm{DTPA}-\mathrm{Gd}$ with the silica thickness of 0 and $5 \mathrm{~nm}$ even showed remarkable 

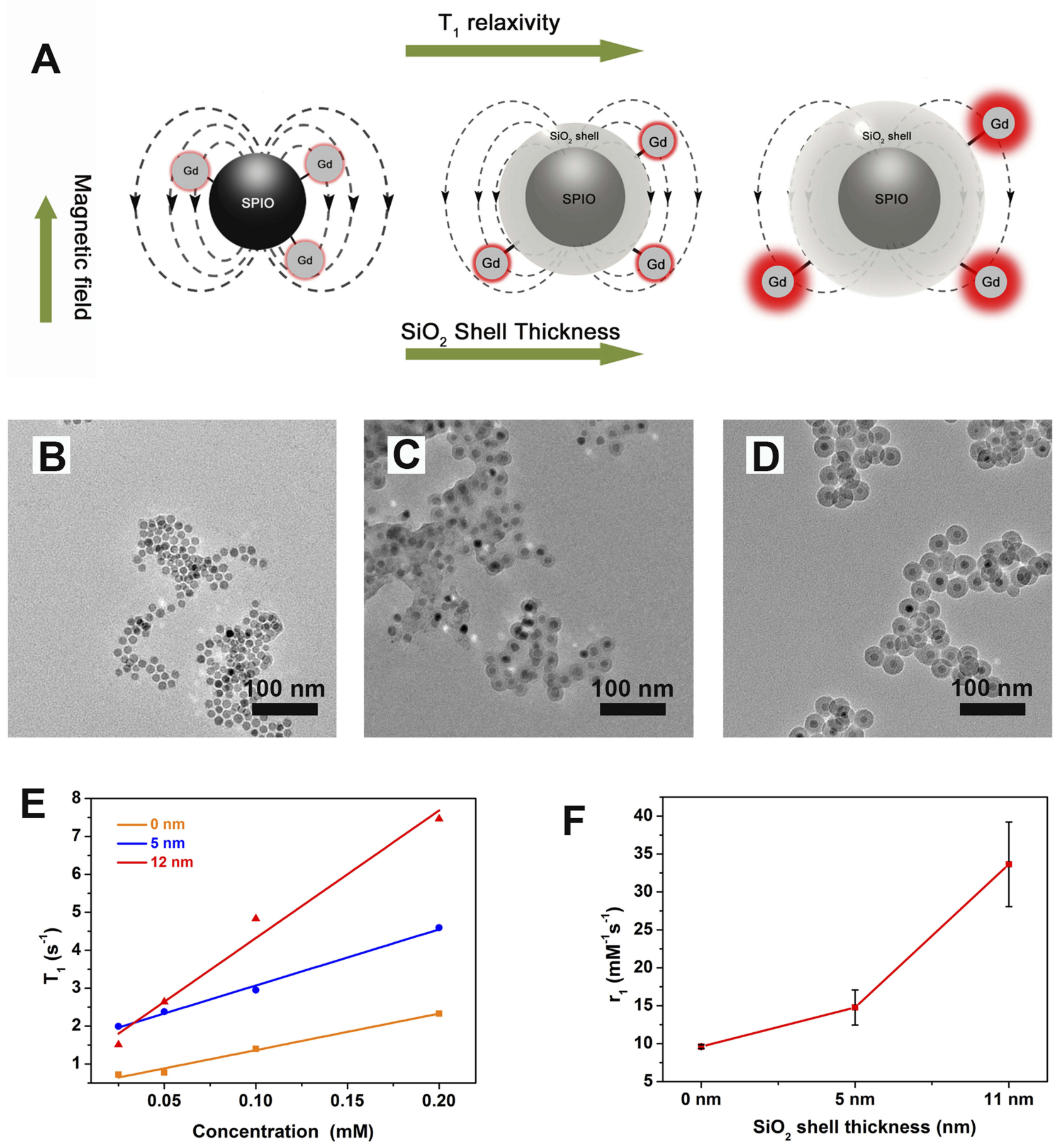

Figure 5 Effect of the silica shell thickness to the $T_{1}$ relaxation of $1 \mathrm{O} @ \mathrm{SiO}_{2}$-DTPA-Gd NPs. (A) schematic cartoons illustrate the Effect of the silica shell thickness to the $T_{1}$ relaxation of $10 @ \mathrm{SiO}_{2}$-DTPA-Gd NPs. TEM images of (B) IO-DTPA-Gd, (C) IO@SiO ${ }_{2}$-DTPA-Gd NPs with the thickness of $5 \mathrm{~nm}$, and (D) IO@SiO 2 -DTPA-Gd NPs with the thickness of $12 \mathrm{~nm}$. (E) $T_{1}$ relaxation of IO-DTPA-Gd, IO@SiO ${ }_{2}$-DTPA-Gd NPs with the thickness of $5 \mathrm{~nm}$, and IO@SiO - -DTPA-Gd NPs with the thickness of $12 \mathrm{~nm}$. (F) $r_{1}$ values change curve of IO-DTPA-Gd, IO@SiO - -DTPA-Gd NPs with the thickness of $5 \mathrm{~nm}$, and IO@SiO 2 -DTPA-Gd NPs with the thickness of $12 \mathrm{~nm}$.

Abbreviations: Gd, gadolinium; IO, iron oxide; TEM, transmission electron microscopy, NPs, nanoparticles.

lower $r_{1}$ value than $\mathrm{SiO}_{2}$-DTPA-Gd. These results could be ascribed to the quenching effect of $T_{2}$ contrast agent to the DTPA-Gd and indicated that the effect of magnetite core to surface DTPA-Gd was decided by the distance. ${ }^{20,36}$ The distance between magnetite and DTPA-Gd was more close, the quenching effect of magnetite to DTPA-Gd was strong enough to hinder the $T_{1}$ relaxation of DTPA-Gd. Along with the increase of distance between magnetite and DTPA-Gd, 
quench effect decreased and showed the enhancement effect of magnetite to DTPA-Gd.

\section{Celluar Imaging And Cytotoxicity Evaluation}

To evaluate the $T_{1}$ contrast ability of $\mathrm{IO} @ \mathrm{SiO}_{2}$-DTPA-Gd NPs on the cellular level, we incubated IO@ $\mathrm{SiO}_{2}$-DTPA-Gd NPs with HeLa cells and conducted $T_{1}$-weighted imaging. It appeared that the cells incubated with $\mathrm{IO} @ \mathrm{SiO}_{2}-\mathrm{DTPA}-\mathrm{Gd}$ NPs showed a remarkable bright signal in $T_{1}$-weighted imaging, which was significantly higher than that of DTPA-Gd treated group (Figure 6A). The signal-to-noise ratio analyses (Figure 6B) further confirmed that IO@ $@ \mathrm{SiO}_{2}$-DTPA-Gd NPs group was much higher than the DTPA-Gd group, demonstrating $\mathrm{IO} @ \mathrm{SiO}_{2}$-DTPA-Gd could be used as a contrast agent to achieve $T_{1}$ contrast imaging. The cytotoxicity of IO@ $\mathrm{SiO}_{2}$-DTPA-Gd NPs was detected via the MTT assay to assess its biocompatibility. It appeared that no obvious decrease in cell viability which could be observed after incubation of IO@ $\mathrm{SiO}_{2}$-DTPA-Gd NPs with Hela cells for $24 \mathrm{~h}$ (Figure S4). Neither apparent agglomeration nor precipitation was observed for $\mathrm{IO} @ \mathrm{SiO}_{2}$-DTPA-Gd NPs after incubation for $24 \mathrm{~h}$ in water and different physiological media including PBS buffer, DMEM culture medium, and blood serum (Figure S5). These results indicated that IO@ $\mathrm{SiO}_{2}$-DTPA-Gd NPs showed the good stability and high biocompatibility, which ensured it as a contrast agent for further biological application.

\section{In Vivo MR Imaging}

Considering the good biocompatibility and high contrast capability of $\mathrm{IO} @ \mathrm{SiO}_{2}$-DTPA-Gd NPs, in vivo MR imaging was then performed for tumor detection. The $T_{1}$-weighted
MR images exhibited that $T_{1}$ signal of tumor region gradually increased and showed sufficient signal to detect tumor in mice (Figure 7A). To quantify the contrast enhancement, the SNR ratio was calculated according to the $T_{1}$-weighted MR images. It appeared that the post-injection signal was much higher than the pre-injection signal (about 1.3 fold at $30 \mathrm{~min}$ ), demonstrating IO@ $\mathrm{SiO}_{2}$-DTPA-Gd could also be used as a contrast agent to achieve in vivo $T_{1}$ contrast imaging (Figure 7B). But due to the lack of modification and tumor targeting module, less accumulation of NPs existed in tumor and resident time was short. Hence, the modification such as PEGylation and tumor targeting module or the tumor microenvironment response module should be added in the further experiment to enhance the retention effect and the visibility of tumor in vivo.

\section{Conclusion}

In conclusion, we developed a high-performance nanosized $T_{1}$ contrast agent IO@ $\mathrm{SiO}_{2}$-DTPA-Gd NPs by loading the Gd-chelate on the surface of silica-coated iron oxide NPs. The synthesized NPs IO@SiO $\mathrm{SiO}_{2}-\mathrm{DTPA}-\mathrm{Gd}$ showed high $r_{1}$ value, which was approximately 6 times higher than Gd-DTPA when the silica shell thickness of $12 \mathrm{~nm}$. In addition, to reveal the underlying mechanism, $\mathrm{IO} @ \mathrm{SiO}_{2}, \quad \mathrm{SiO}_{2}-\mathrm{DTPA}-\mathrm{Gd}$, and $\mathrm{IO} @ \mathrm{SiO}_{2}-\mathrm{DTPA}-\mathrm{Gd}$ were synthesized respectively and compared to each other. These results clearly indicated that the improved $T_{1}$ relaxation of $\mathrm{IO} @ \mathrm{SiO}_{2}-\mathrm{DTPA}-\mathrm{Gd}$ NPs was not only caused by the increased molecular tumbling time but also caused by the magnetite core of $\mathrm{IO} @ \mathrm{SiO}_{2}$. Additionally, the increase of distance between magnetite core and DTPA-Gd could also reduce the quenching effect and
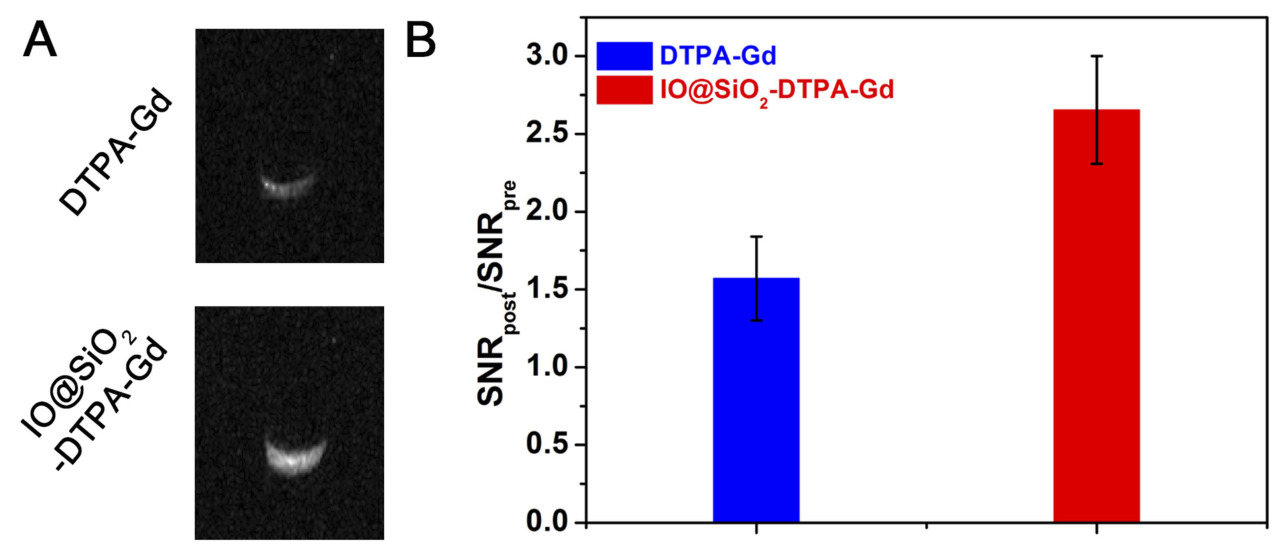

Figure 6 Cellular level imaging. (A) $T_{1}$-weighted MR images of cells incubated with DTPA-Gd and IO@SiO 2 -DTPA-Gd NPs. (B) Signal-to-noise ratio changes of $T_{1}$-weighted images of Hela cells incubated with DTPA-Gd and IO@SiO 2 -DTPA-Gd NPs.

Abbreviations: Gd, gadolinium; IO, iron oxide, NPs, nanoparticles. 
A

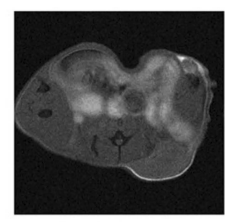

Pre-injection

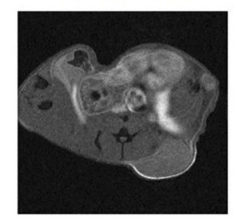

$45 \mathrm{~min}$

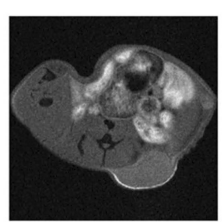

$15 \mathrm{~min}$

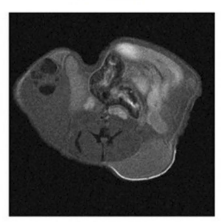

$60 \mathrm{~min}$

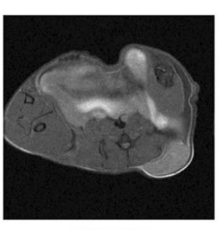

$30 \mathrm{~min}$

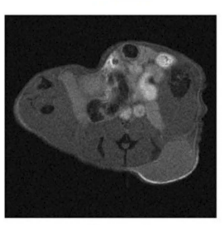

$120 \mathrm{~min}$
B

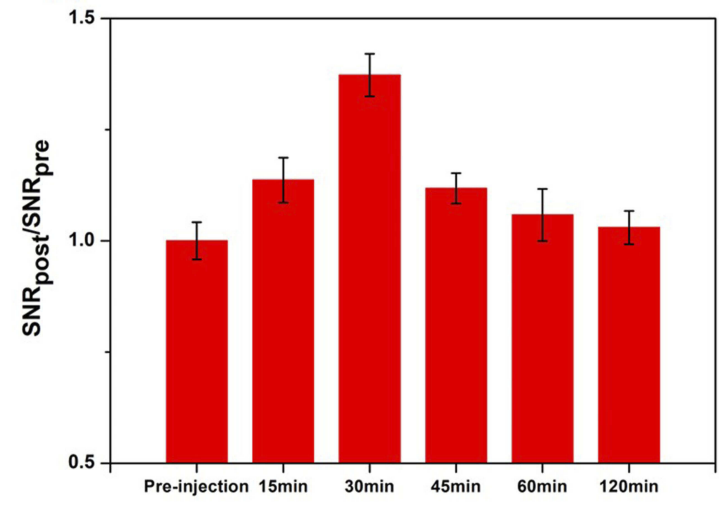

Figure 7 In vivo MR imaging. (A) $T_{1}$-weighted MR images of tumor at pre-injection and I5, 30, 45, 60 and I20 min after intravenous injection of IO@SiO respectively (B) Signal-to-noise ratio changes of $T_{1}$-weighted images of tumor at different time after administration of IO@SiO 2 -DTPA-Gd NPs.

Abbreviations: Gd, gadolinium; IO, iron oxide, NPs, nanoparticles.

show the enhancement effect of magnetite core to DTPAGd. Moreover, the remarkable bright signal in $T_{1^{-}}$ weighted imaging of IO@ $\mathrm{SiO}_{2}$-DTPA-Gd NPs incubating cells and in vivo MR imaging indicated that $\mathrm{IO} @ \mathrm{SiO}_{2^{-}}$ DTPA-Gd NPs could be the potential MRI contrast agent for the future clinical application.

\section{Acknowledgments}

This work was supported by the National Natural Science Foundation of China (NSFC) (Grant Nos. 81571660 and 81601607) and the National Natural Science Foundation of China (NSFC) (Grant No. 81871421).

\section{Disclosure}

The authors report no conflicts of interest in this work.

\section{References}

1. Shin T-H, Choi Y, Kim S, Cheon J. Recent advances in magnetic nanoparticle-based multi-modal imaging. Chem Soc Rev. 2015;44 (14):4501-4516. doi:10.1039/c4cs00345d

2. Fan W, Yung B, Huang P, Chen X. Nanotechnology for multimodal synergistic cancer therapy. Chem Rev. 2017;117(22):13566-13638. doi:10.1021/acs.chemrev.7b00258

3. Johnson NJJ, He S, Nguyen Huu VA, Almutairi A. Compact micellization: a strategy for Ultrahigh T1 magnetic resonance contrast with gadolinium-based nanocrystals. ACS Nano. 2016;10(9):8299-8307. doi:10.1021/acsnano.6b02559

4. Qin L, Sun Z-Y, Cheng K, et al. Zwitterionic manganese and gadolinium metal-organic frameworks as efficient contrast agents for in vivo magnetic resonance imaging. ACS Appl Mater Interfaces. 2017;9 (47):41378-41386. doi:10.1021/acsami.7b09608

5. Peng Y-K, Lui CNP, Chen Y-W, et al. Engineering of single magnetic particle carrier for living brain cell imaging: a tunable T1-/T2-/dualmodal contrast agent for magnetic resonance imaging application. Chem Mater. 2017;29(10):4411-4417. doi:10.1021/acs.chemmater.7b 00884
6. Zhao Z, Chi X, Yang L, et al. Cation exchange of anisotropic-shaped magnetite nanoparticles generates high-relaxivity contrast agents for liver tumor imaging. Chem Mater. 2016;28(10):3497-3506. doi:10. 1021/acs.chemmater.6b01256

7. Zhao Z, Zhou Z, Bao J, et al. Octapod iron oxide nanoparticles as high-performance $\mathrm{T}_{2}$ contrast agents for magnetic resonance imaging. Nat Commun. 2013;4:2266. doi:10.1038/ncomms3266

8. Na HB, Lee JH, An K, et al. Development of a T1 contrast agent for magnetic resonance imaging using $\mathrm{MnO}$ nanoparticles. Angew ChemInt Ed. 2007;119(28):5493-5497. doi:10.1002/ange.200604775

9. Zhao Z, Wang X, Zhang Z, et al. Real-time monitoring of arsenic trioxide release and delivery by activatable T1 imaging. ACS Nano. 2015;9(3):2749-2759. doi:10.1021/nn506640h

10. Zhao Z, Bao J, Fu C, Lei M, Cheng J. Controllable synthesis of manganese oxide nanostructures from $0-\mathrm{D}$ to $3-\mathrm{D}$ and mechanistic investigation of internal relation between structure and T1 relaxivity. Chem Mater. 2017;29(24):10455-10468. doi:10.1021/acs.chemmater.7b04100

11. Lei M, Fu C, Cheng X, et al. Activated surface charge-reversal manganese oxide nanocubes with high surface-to-volume ratio for accurate magnetic resonance tumor imaging. Adv Funct Mater. 2017;27(30):1700978. doi:10.1002/adfm.201700978

12. Werner EJ, Datta A, Jocher CJ, Raymond KN. High-relaxivity MRI contrast agents: where coordination chemistry meets medical imaging. Angew Chem-Int Ed. 2008;47(45):8568-8580. doi:10.1002/anie. 200800212

13. Ni K, Zhao Z, Zhang Z, et al. Geometrically confined ultrasmall gadolinium oxide nanoparticles boost the T1 contrast ability. Nanoscale. 2016;8(6):3768-3774. doi:10.1039/c5nr08402d

14. Ananta JS, Godin B, Sethi R, et al. Geometrical confinement of gadolinium-based contrast agents in nanoporous particles enhances T1 contrast. Nat Nanotechnol. 2010;5:815. doi:10.1038/nnano.2010.203

15. Paik T, Tr G, Am P, Yun H, Cb M. Designing tripodal and triangular gadolinium oxide nanoplates and self-assembled nanofibrils as potential multimodal bioimaging probes. ACS Nano. 2013;7(3):28502859. doi: $10.1021 / \mathrm{nn} 4004583$

16. Li F, Li Z, Jin X, et al. Ultra-small gadolinium oxide nanocrystal sensitization of non-small-cell lung cancer cells toward X-ray irradiation by promoting cytostatic autophagy. Int $J$ Nanomedicine. 2019;14:2415-2431. doi:10.2147/IJN.S193676

17. Bae KH, Kim YB, Lee Y, Hwang J, Park H, Park TG. Bioinspired synthesis and characterization of gadolinium-labeled magnetite nanoparticles for dual contrast T1- and T2-weighted magnetic resonance imaging. Bioconjugate Chem. 2010;21(3):505-512. doi:10.1021/bc9 00424u 
18. Yang H, Zhuang Y, Sun Y, et al. Targeted dual-contrast T1- and T2weighted magnetic resonance imaging of tumors using multifunctional gadolinium-labeled superparamagnetic iron oxide nanoparticles. Biomaterials. 2011;32(20):4584-4593. doi:10.1016/j.biomaterials.2011. 03.018

19. Li F, Zhi D, Luo Y, et al. Core/shell Fe3O4/Gd2O3 nanocubes as T1T2 dual modal MRI contrast agents. Nanoscale. 2016;8(25):1282612833. doi:10.1039/c6nr02620f

20. J-s C, Lee J-H, Shin T-H, Song H-T, Kim EY, Cheon J. Self-confirming "AND" logic nanoparticles for fault-free MRI. J Am Chem Soc. 2010;132(32):11015-11017. doi:10.1021/ja104503g

21. Zhu X, Lin H, Wang L, et al. Activatable T1 relaxivity recovery nanoconjugates for kinetic and sensitive analysis of matrix metalloprotease 2. ACS Appl Mater Interfaces. 2017;9(26):21688-21696. doi:10.1021/acsami.7b05389

22. J-s C, Kim S, Yoo D, et al. Distance-dependent magnetic resonance tuning as a versatile MRI sensing platform for biological targets. Nat Mater. 2017;16:537. doi:10.1038/nmat4846

23. Gallo J, Harriss BI, Hernández-Gil J, Bañobre-López M, Long NJ. Probing T1-T2 interactions and their imaging implications through a thermally responsive nanoprobe. Nanoscale. 2017;9(31):1131811326. doi:10.1039/c7nr01733b

24. Keasberry NA, Bañobre-López M, Wood C, Stasiuk GJ, Gallo J, Long NJ. Tuning the relaxation rates of dual-mode T1/T2 nanoparticle contrast agents: a study into the ideal system. Nanoscale. 2015;7 (38):16119-16128. doi:10.1039/c5nr04400f

25. Zhou Z, Huang D, Bao J, et al. A synergistically enhanced T1-T2 dual-modal contrast agent. Adv Mater. 2012;24(46):6223-6228. doi:10.1002/adma.201203169

26. Santra S, Jativa SD, Kaittanis C, Normand G, Grimm J, Perez JM. Gadolinium-encapsulating iron oxide nanoprobe as activatable NMR/ MRI contrast agent. ACS Nano. 2012;6(8):7281-7294. doi:10.1021/ nn302393e
27. He Q, Shi J. MSN anti-cancer nanomedicines: chemotherapy enhancement, overcoming of drug resistance, and metastasis inhibition. Adv Mater. 2014;26(3):391-411. doi:10.1002/adma.201303123

28. Chen Y, Chen H, Zeng D, et al. Core/shell structured hollow mesoporous nanocapsules: a potential platform for simultaneous cell imaging and anticancer drug delivery. ACS Nano. 2010;4(10):60016013. doi: $10.1021 / \mathrm{nn} 1015117$

29. Cao YC. Synthesis of square gadolinium-oxide nanoplates. $J \mathrm{Am}$ Chem Soc. 2004;126(24):7456-7457. doi:10.1021/ja0481676

30. H1 D, Yx Z, Wang S, Jm X, Sc X, Gh L. Fe3O4@SiO2 Core/shell nanoparticles: the silica coating regulations with a single core for different core sizes and shell thicknesses. Chem Mater. 2012;24 (23):4572-4580. doi:10.1021/cm302828d

31. Yi DK, Lee SS, Papaefthymiou GC, Ying JY. Nanoparticle architectures templated by $\mathrm{SiO} 2 / \mathrm{Fe} 2 \mathrm{O} 3$ nanocomposites. Chem Mater. 2006;18(3):614-619. doi:10.1021/cm0512979

32. Sato T, Shimosato T, Klinman DM. Silicosis and lung cancer: current perspectives. Lung Cancer. 2018;9:91-101. doi:10.2147/LCTT. S 156376

33. Das M, Yi DK, An SS. Analyses of protein corona on bare and silicacoated gold nanorods against four mammalian cells. Int $J$ Nanomedicine. 2015;10:1521-1545. doi:10.2147/IJN.S76187

34. Wang L, Lin H, Ma L, et al. Albumin-based nanoparticles loaded with hydrophobic gadolinium chelates as T1-T2 dual-mode contrast agents for accurate liver tumor imaging. Nanoscale. 2017;9 (13):4516-4523. doi:10.1039/c7nr01134b

35. Wang L, Zhu X, Tang $X$, et al. A multiple gadolinium complex decorated fullerene as A highly sensitive T1 contrast agent. Chem Commun. 2015;51(21):4390-4393. doi:10.1039/C5CC00285K

36. Shin T-H, J-s C, Yun S, et al. T1 and T2 dual-mode MRI contrast agent for enhancing accuracy by engineered nanomaterials. ACS Nano. 2014;8(4):3393-3401. doi:10.1021/nn405977t
International Journal of Nanomedicine

\section{Publish your work in this journal}

The International Journal of Nanomedicine is an international, peerreviewed journal focusing on the application of nanotechnology in diagnostics, therapeutics, and drug delivery systems throughout the biomedical field. This journal is indexed on PubMed Central, MedLine, CAS, SciSearch ${ }^{\mathbb{R}}$, Current Contents ${ }^{\mathbb{B}} /$ Clinical Medicine,
Journal Citation Reports/Science Edition, EMBase, Scopus and the Elsevier Bibliographic databases. The manuscript management system is completely online and includes a very quick and fair peer-review system, which is all easy to use. Visit http://www.dovepress.com/ testimonials.php to read real quotes from published authors. 